Bol. Soc. Esp. Mat. Apl.

$n^{\circ} 51(2010), 55-63$

\title{
PLANAR BIMODAL PIECEWISE LINEAR SYSTEMS. BIFURCATION DIAGRAMS
}

\author{
J. FERRER, M. D. MAGRET, J. R. PACHA AND M. PEÑA \\ Dept. de Matemàtica Aplicada I, UPC \\ Av. Diagonal, 647 08028, Barcelona \\ \{josep.ferrer,m.dolors .magret, juan.ramon. pacha, marta . penya\}@upc.edu
}

\begin{abstract}
The set of planar bimodal linear control systems is partitioned into a finite number of differentiable strata, each of them consisting of those systems having canonical forms (for the equivalence relation which corresponds to admissible changes of basis) differing only in the values of the continuous invariants. Bifurcation diagrams with regard to this stratification are derived.
\end{abstract}

Key words: Canonical form, stratification, bifurcation diagram.

AMS subject classifications: $\quad$ 93B10, 93B27, 93C10

\section{Introduction}

Piecewise linear systems have attracted the interest of researchers because of their interesting dynamical properties and the wide range of applications. The most common piecewise linear systems found in practice are in two or three dimensions. See for example [3], [4], [5].

In this paper, we tackle bifurcation diagrams for planar bimodal piecewise control systems. We consider $2 D$ control linear systems acting on complementary half-planes and the equivalence relation defined by basis changes, preserving continuity along a given line ("admissible basis changes"). As the set of equivalence classes is not locally finite, we consider the union of equivalence classes differing only in the continuous invariants in the canonical form under this equivalence relation found in [6]. There are a finite number of sets in this partition, each of them is proved to be a differentiable manifold, therefore constitutes a finite stratification of the space of systems. This is the starting point to obtain bifurcation diagrams, with regard to this classification. Moreover, canonical forms can be applied to study controllability and other dynamical properties in each stratum.

In section 2, we state the definitions of bimodal piecewise linear systems and admissible basis changes. In section 3 , we recall the canonical forms for order two bimodal systems. In section 4, we stratify the set of triples of matrices 
defining order two bimodal systems. Finally, in section 5, we show a bifurcation diagram.

Throughout the paper, $\mathbb{R}$ will denote the set of real numbers, $M_{n \times m}(\mathbb{R})$ the set of matrices with $m$ rows and $n$ columns (in the particular case where $m=n$ we will denote the set simply by $\left.M_{n}(\mathbb{R})\right), G l_{n}(\mathbb{R})$ the set of all invertible matrices in $M_{n}(\mathbb{R})$ and by $\left(e_{1}, \ldots, e_{n}\right)$ the natural basis of the Euclidian space $\mathbb{R}^{n}$.

\section{Bimodal Piecewise Linear Systems}

Bimodal piecewise linear systems consist of two linear dynamics acting on each side of a given hyperplane. Most of elementary non-linear circuits found in practice may be modeled with two linear regions separated by parallel boundaries hyperplanes, with two or three state variables. See [3], [4], [7], [8], where different topics about these systems are studied.

Bimodal (piecewise) linear systems can be defined by two control linear systems:

$$
\left\{\begin{array}{l}
\dot{\boldsymbol{x}}(t)=A_{1} \boldsymbol{x}(t)+B_{1}, \\
\boldsymbol{y}(t)=C \boldsymbol{x}(t),
\end{array} \quad \text { if } \boldsymbol{y}(t) \leq 0, \quad\left\{\begin{array}{l}
\dot{\boldsymbol{x}}(t)=A_{2} \boldsymbol{x}(t)+B_{2}, \\
\boldsymbol{y}(t)=C \boldsymbol{x}(t),
\end{array} \quad \text { if } \mathbf{y}(t) \geq 0\right.\right.
$$

where $A_{1}, A_{2} \in M_{n}(\mathbb{R}) ; B_{1}, B_{2} \in M_{n \times 1}(\mathbb{R}) ; C \in M_{1 \times n}(\mathbb{R})$, being the dynamics continuous along a separating hyperplane $C \boldsymbol{x}=0$ for some matrix $C \in M_{1 \times n}(\mathbb{R})$. For simplicity, we will consider $C=\left(\begin{array}{lll}1 & 0 \ldots 0\end{array}\right) M_{1 \times n}(\mathbb{R})$ and that the dynamics is continuous along the hyperplane $H=\left\{\boldsymbol{x} \in \mathbb{R}^{n}: C \boldsymbol{x}=0\right\}$, and hence: $H=\left\{\boldsymbol{x} \in \mathbb{R}^{n}: x_{1}=0\right\}$.

Then continuity along $H$ is equivalent to:

$$
B_{2}=B_{1}, \quad A_{2} e_{i}=A_{1} e_{i}, \quad 2 \leq i \leq n .
$$

We will simply write $B=B_{1}=B_{2}$. Thus any bimodal piecewise linear system can be defined by a triple of matrices $\left(A_{1}, A_{2}, B\right)$, where $A_{1}, A_{2}$ differ only in the first column.

Notation Throughout the paper, $\mathcal{X}$ will denote the set of triples of matrices defining bimodal piecewise linear systems,

$$
\mathcal{X}=\left\{\left(A_{1}, A_{2}, B\right) \in M_{n}(\mathbb{R}) \times M_{n}(\mathbb{R}) \times M_{n \times 1}(\mathbb{R}) \mid A_{2} e_{i}=A_{1} e_{i}, 2 \leq i \leq n\right\}
$$

which is obviously a differentiable manifold (of dimension $n^{2}+2 n$ ).

As in [6], we consider basis changes preserving the hiperplanes $x_{1}(t)=k$ in order to allow the results below to be also applied in the cases where a separating hyperplane $x_{1}(t)=\delta, \delta \neq 0$, are considered (see, for example, [3]).

Definition 1 Basis changes in the state variables space preserving the hyperplanes $x_{1}(t)=k$ will be called admissible basis changes. Thus, they are basis changes given by a matrix $S \in G l_{n}(\mathbb{R})$,

$$
S=\left(\begin{array}{cc}
1 & 0 \\
U & T
\end{array}\right), \quad T \in G l_{n-1}(\mathbb{R}) .
$$


Let us denote by $\mathcal{S}$ the Lie subgroup of $G l_{n}(\mathbb{R})$

$$
\mathcal{S}:=\left\{S \in G l_{n}(\mathbb{R}) \mid S=\left(\begin{array}{cc}
1 & 0 \\
U & T
\end{array}\right), T \in G l_{n-1}(\mathbb{R})\right\}
$$

We consider the equivalence relation in the set of matrices $\mathcal{X}$ which corresponds to admissible basis changes.

Definition 2 Two triples of matrices $\left(A_{1}, A_{2}, B\right),\left(A^{\prime}{ }_{1}, A^{\prime}{ }_{2}, B^{\prime}\right) \in \mathcal{X}$ are said to be equivalent if there exists a matrix $S \in \mathcal{S}$ (representing an admissible basis change) such that $\left(A^{\prime}{ }_{1}, A^{\prime}{ }_{2}, B^{\prime}\right)=\left(S^{-1} A_{1} S, S^{-1} A_{2} S, S^{-1} B\right)$.

This equivalence relation partitions $\mathcal{X}$ into finer equivalence classes than the similarity equivalence relation.

\section{Canonical forms for $n=2$}

A canonical form is a representative in each equivalence class which is easier to deal with, and therefore calculations become simpler using it. In [3], canonical forms were obtained, assuming observability. In [6] canonical forms in the nonobservable case are obtained, in the case where the observability matrix of the system rank equal to $n-1$. In particular, in the case $n=2$ these canonical forms and the matrices $S$ which correspond to admissible basis changes are listed below. We will use ( $\mathrm{CFN}), \mathrm{N}=1,2, \ldots$ to label them.

Let us consider a triple of matrices defining an order two bimodal system

$$
\left(\left(\begin{array}{ll}
a_{1} & a_{3} \\
a_{2} & a_{4}
\end{array}\right),\left(\begin{array}{ll}
\gamma_{1} & a_{3} \\
\gamma_{2} & a_{4}
\end{array}\right),\left(\begin{array}{l}
b_{1} \\
b_{2}
\end{array}\right)\right) .
$$

Let us assume that the system is observable $\left(a_{3} \neq 0\right)$. Then (see [3]), the corresponding canonical forms $A_{1}^{c}, A_{2}^{c}, B^{c}$ for the matrices $A_{1}, A_{2}$ and $B$ respectively, are:

- $\underline{\text { Case 0: }} a_{3} \neq 0$,

$$
\begin{aligned}
& A_{1}^{c}=\left(\begin{array}{cc}
a_{1}+a_{4} & 1 \\
a_{2} a_{3}-a_{1} a_{4} & 0
\end{array}\right)=\left(\begin{array}{cc}
\operatorname{tr} A_{1} & 1 \\
\operatorname{det} A_{1} & 0
\end{array}\right), \\
& A_{2}^{c}=\left(\begin{array}{cc}
\gamma_{1}+a_{4} & 1 \\
a_{3} \gamma_{2}-a_{4} \gamma_{1} & 0
\end{array}\right)=\left(\begin{array}{cc}
\operatorname{tr} A_{2} & 1 \\
\operatorname{det} A_{2} & 0
\end{array}\right), \\
& B^{c}=\left(\begin{array}{c}
b_{1} \\
a_{3} b_{2}-a_{4} b_{1}
\end{array}\right) ; S=\left(\begin{array}{cc}
1 & 0 \\
\frac{a_{4}}{a_{3}} & \frac{1}{a_{3}}
\end{array}\right) .
\end{aligned}
$$

From now on, we will assume that the system is unobservable: $a_{3}=0$. We distinguish several cases.

- $\underline{\text { Case 1: }} a_{3}=0, a_{1} \neq a_{4}, \gamma_{1} \neq a_{4}$. 
- If $\gamma_{2}=a_{2} \frac{a_{4}-\gamma_{1}}{a_{4}-a_{1}}, b_{2}+\frac{a_{2} b_{1}}{a_{4}-a_{1}}=0$,

$$
\begin{aligned}
A_{1}^{c} & =\left(\begin{array}{cc}
a_{1} & 0 \\
0 & a_{4}
\end{array}\right), A_{2}^{c}=\left(\begin{array}{cc}
\gamma_{1} & 0 \\
0 & a_{4}
\end{array}\right), B^{c}=\left(\begin{array}{c}
b_{1} \\
0
\end{array}\right) ; \\
S & =\left(\begin{array}{cc}
1 & 0 \\
-\frac{a_{2}}{a_{4}-a_{1}} & t
\end{array}\right), \text { for any } t \neq 0 .
\end{aligned}
$$

- If $\gamma_{2}=a_{2} \frac{a_{4}-\gamma_{1}}{a_{4}-a_{1}}, b_{2}+\frac{a_{2} b_{1}}{a_{4}-a_{1}} \neq 0$,

$$
\begin{aligned}
A_{1}^{c} & =\left(\begin{array}{cc}
a_{1} & 0 \\
0 & a_{4}
\end{array}\right), A_{2}^{c}=\left(\begin{array}{cc}
\gamma_{1} & 0 \\
0 & a_{4}
\end{array}\right), B^{c}=\left(\begin{array}{c}
b_{1} \\
1
\end{array}\right) ; \\
S & =\left(\begin{array}{cc}
1 & 0 \\
-\frac{a_{2}}{a_{4}-a_{1}} & b_{2}+b_{1} \frac{a_{2}}{a_{4}-a_{1}}
\end{array}\right) .
\end{aligned}
$$

- If $\gamma_{2} \neq a_{2} \frac{a_{4}-\gamma_{1}}{a_{4}-a_{1}}$

$$
\begin{aligned}
& A_{1}^{c}=\left(\begin{array}{cc}
a_{1} & 0 \\
0 & a_{4}
\end{array}\right), A_{2}^{c}=\left(\begin{array}{cc}
\gamma_{1} & 0 \\
1 & a_{4}
\end{array}\right), B^{c}=\left(\begin{array}{c}
b_{1} \\
\frac{b_{2}+b_{1} \frac{a_{2}}{a_{4}-a_{1}}}{\gamma_{2}-a_{2} \frac{a_{4}-\gamma_{1}}{a_{4}-a_{1}}}
\end{array}\right) ; \\
& S=\left(\begin{array}{cc}
1 & 0 \\
-\frac{a_{2}}{a_{4}-a_{1}} & \gamma_{2}-a_{2} \frac{a_{4}-\gamma_{1}}{a_{4}-a_{1}}
\end{array}\right) .
\end{aligned}
$$

- $\underline{\text { Case 2: }} a_{3}=0, a_{1}=a_{4}, \gamma_{1} \neq a_{4}$.

- If $a_{2}=0, b_{2}+\frac{\gamma_{2} b_{1}}{a_{4}-\gamma_{1}}=0$,

$$
\begin{aligned}
A_{1}^{c} & =\left(\begin{array}{cc}
a_{4} & 0 \\
0 & a_{4}
\end{array}\right), A_{2}^{c}=\left(\begin{array}{cc}
\gamma_{1} & 0 \\
0 & a_{4}
\end{array}\right), B^{c}=\left(\begin{array}{c}
b_{1} \\
0
\end{array}\right) ; \\
S & =\left(\begin{array}{cc}
1 & 0 \\
-\frac{\gamma_{2}}{a_{4}-\gamma_{1}} & t
\end{array}\right) \text { for any } \mathrm{t} \neq 0 .
\end{aligned}
$$

- If $a_{2}=0, b_{2}+\frac{\gamma_{2} b_{1}}{a_{4}-\gamma_{1}} \neq 0$,

$$
\begin{aligned}
A_{1}^{c} & =\left(\begin{array}{cc}
a_{4} & 0 \\
0 & a_{4}
\end{array}\right), A_{2}^{c}=\left(\begin{array}{cc}
\gamma_{1} & 0 \\
0 & a_{4}
\end{array}\right), B^{c}=\left(\begin{array}{c}
b_{1} \\
1
\end{array}\right) ; \\
S & =\left(\begin{array}{cc}
1 & 0 \\
-\frac{\gamma_{2}}{a_{4}-\gamma_{1}} & b_{2}+b_{1} \frac{\gamma_{2}}{a_{4}-\gamma_{1}}
\end{array}\right) .
\end{aligned}
$$

- If $a_{2} \neq 0$,

$$
\begin{aligned}
A_{1}^{c} & =\left(\begin{array}{cc}
a_{4} & 0 \\
1 & a_{4}
\end{array}\right), A_{2}^{c}=\left(\begin{array}{cc}
\gamma_{1} & 0 \\
0 & a_{4}
\end{array}\right), B^{c}=\left(\frac{1}{a_{2}}\left[b_{2}+b_{1} \frac{\gamma_{2}}{a_{4}-\gamma_{1}}\right]\right) ; \\
S & =\left(\begin{array}{cc}
1 & 0 \\
-\frac{\gamma_{2}}{a_{4}-\gamma_{1}} & a_{2}
\end{array}\right) .
\end{aligned}
$$


- $\underline{\text { Case 3: }} a_{3}=0, a_{1} \neq a_{4}, \gamma_{1}=a_{4}$.

- If $\gamma_{2}=0, b_{2}=0$,

$$
\begin{aligned}
A_{1}^{c} & =\left(\begin{array}{cc}
a_{1} & 0 \\
0 & a_{4}
\end{array}\right), A_{2}^{c}=\left(\begin{array}{cc}
a_{4} & 0 \\
0 & a_{4}
\end{array}\right), B^{c}=\left(\begin{array}{c}
b_{1} \\
0
\end{array}\right) ; \\
S & =\left(\begin{array}{cc}
1 & 0 \\
-\frac{a_{2}}{a_{4}-a_{1}} & t
\end{array}\right), \text { for any } t \neq 0 .
\end{aligned}
$$

- If $a_{2}=0, b_{2} \neq 0$,

$$
\begin{aligned}
A_{1}^{c} & =\left(\begin{array}{cc}
a_{1} & 0 \\
0 & a_{4}
\end{array}\right), A_{2}^{c}=\left(\begin{array}{cc}
a_{4} & 0 \\
0 & a_{4}
\end{array}\right), B^{c}=\left(\begin{array}{c}
b_{1} \\
1
\end{array}\right) ; \\
S & =\left(\begin{array}{cc}
1 & 0 \\
-\frac{a_{2}}{a_{4}-a_{1}} & b_{2}
\end{array}\right) .
\end{aligned}
$$

- If $a_{2} \neq 0$,

$$
\begin{aligned}
A_{1}^{c} & =\left(\begin{array}{cc}
a_{1} & 0 \\
0 & a_{4}
\end{array}\right), A_{2}^{c}=\left(\begin{array}{cc}
a_{4} & 0 \\
1 & a_{4}
\end{array}\right), B^{c}=\left(\frac{1}{\gamma_{2}}\left[b_{2}+b_{1} \frac{a_{2}}{a_{4}-a_{1}}\right]\right) ; \\
S & =\left(\begin{array}{cc}
1 & 0 \\
-\frac{a_{2}}{a_{4}-a_{1}} & \gamma_{2}
\end{array}\right) .
\end{aligned}
$$

- $\underline{\text { Case 4: }} a_{3}=0, a_{1}=a_{4}=\gamma_{1}$.

- If $a_{2}=0, \gamma_{2}=0, b_{1} \neq 0$,

$$
\begin{aligned}
A_{1}^{c} & =\left(\begin{array}{cc}
a_{4} & 0 \\
0 & a_{4}
\end{array}\right), A_{2}^{c}=\left(\begin{array}{cc}
a_{4} & 0 \\
0 & a_{4}
\end{array}\right), B^{c}=\left(\begin{array}{c}
b_{1} \\
0
\end{array}\right) ; \\
S & =\left(\begin{array}{cc}
1 & 0 \\
\frac{b_{2}}{b_{1}} & t
\end{array}\right) \text { for any } t \neq 0 .
\end{aligned}
$$

- If $a_{2}=0, \gamma_{2}=0, b_{1}=0, b_{2}=0$,

$$
\begin{aligned}
A_{1}^{c} & =\left(\begin{array}{cc}
a_{4} & 0 \\
0 & a_{4}
\end{array}\right), A_{2}^{c}=\left(\begin{array}{cc}
a_{4} & 0 \\
0 & a_{4}
\end{array}\right), B^{c}=\left(\begin{array}{l}
0 \\
0
\end{array}\right) ; \\
S & =\left(\begin{array}{cc}
1 & 0 \\
u & t
\end{array}\right), \text { for any } t \neq 0, u .
\end{aligned}
$$

- If $a_{2}=0, \gamma_{2}=0, b_{1}=0, b_{2} \neq 0$,

$$
\begin{aligned}
A_{1}^{c} & =\left(\begin{array}{cc}
a_{4} & 0 \\
0 & a_{4}
\end{array}\right), A_{2}^{c}=\left(\begin{array}{cc}
a_{4} & 0 \\
0 & a_{4}
\end{array}\right), B^{c}=\left(\begin{array}{l}
0 \\
1
\end{array}\right) ; \\
S & =\left(\begin{array}{cc}
1 & 0 \\
u & b_{2}
\end{array}\right) \text { for any } u .
\end{aligned}
$$


- If $a_{2}=0, \gamma_{2} \neq 0, b_{1} \neq 0$,

$$
\begin{aligned}
A_{1}^{c} & =\left(\begin{array}{cc}
a_{4} & 0 \\
0 & a_{4}
\end{array}\right), A_{2}^{c}=\left(\begin{array}{cc}
a_{4} & 0 \\
1 & a_{4}
\end{array}\right), B^{c}=\left(\begin{array}{c}
b_{1} \\
0
\end{array}\right) ; \\
S & =\left(\begin{array}{cc}
1 & 0 \\
\frac{b_{2}}{b_{1}} & \gamma_{2}
\end{array}\right) .
\end{aligned}
$$

- If $a_{2}=0, \gamma_{2} \neq 0, b_{1}=0$,

$$
\begin{aligned}
A_{1}^{c} & =\left(\begin{array}{cc}
a_{4} & 0 \\
0 & a_{4}
\end{array}\right), A_{2}^{c}=\left(\begin{array}{cc}
a_{4} & 0 \\
1 & a_{4}
\end{array}\right), B^{c}=\left(\begin{array}{c}
0 \\
\frac{b_{2}}{\gamma_{2}}
\end{array}\right) ; \\
S & =\left(\begin{array}{cc}
1 & 0 \\
u & \gamma_{2}
\end{array}\right) \text { for any } u .
\end{aligned}
$$

- If $a_{2} \neq 0, b_{1} \neq 0$,

$$
\begin{aligned}
A_{1}^{c} & =\left(\begin{array}{cc}
a_{4} & 0 \\
1 & a_{4}
\end{array}\right), A_{2}^{c}=\left(\begin{array}{cc}
a_{4} & 0 \\
\frac{\gamma_{2}}{a_{2}} & a_{4}
\end{array}\right), B^{c}=\left(\begin{array}{c}
b_{1} \\
0
\end{array}\right) \\
S & =\left(\begin{array}{cc}
1 & 0 \\
\frac{b_{2}}{b_{1}} & a_{2}
\end{array}\right) .
\end{aligned}
$$

- If $a_{2} \neq 0, b_{1}=0$,

$$
\begin{aligned}
A_{1}^{c} & =\left(\begin{array}{cc}
a_{4} & 0 \\
1 & a_{4}
\end{array}\right), A_{2}^{c}=\left(\begin{array}{cc}
a_{4} & 0 \\
\frac{\gamma_{2}}{a_{2}} & a_{4}
\end{array}\right), B^{c}=\left(\begin{array}{c}
0 \\
\frac{b_{2}}{a_{2}}
\end{array}\right) ; \\
S & =\left(\begin{array}{cc}
1 & 0 \\
u & a_{2}
\end{array}\right), \text { for any } u .
\end{aligned}
$$

\section{Stratification}

A finite partition of the differentiable manifold $\mathcal{X}$ may be deduced from that in equivalence classes: consider the sets consisting of all equivalence classes with canonical forms of the "same type", but with different values for the parameters. The sets thus obtained are disjoint sets and, as we will show, differentiable manifolds. Therefore, they constitute a stratification of $\mathcal{X}$.

In order to use Arnold's techniques (see [1]), the starting point is that equivalence classes are the orbits of the Lie group action of $\mathcal{S}$ on $\mathcal{X}$ defined by $\alpha\left(S,\left(A_{1}, A_{2}, B\right)\right)=\left(S^{-1} A_{1} S, S^{-1} A_{2} S, S^{-1} B\right)$.

Given $\left(A_{1}, A_{2}, B\right) \in \mathcal{X}$, we will denote by $\mathcal{O}\left(A_{1}, A_{2}, B\right)$ its orbit and consider the partition of $\mathcal{X}$ into sets, each of them corresponding to the union of orbits or equivalence classes having associated a canonical form of the same type; namely, $E_{1}$ is the set of all triples of matrices having canonical form of type CF1, $E_{2}$ the set of all those having canonical form of type CF2, and so on. Note that these orbits are differentiable manifolds (see [9]).

Theorem 1 The sets $E_{i}, i=1, \ldots, 17$ are differentiable manifolds. 
Proof. $E_{i}, i \neq 2,5,8$ are open sets of linear varieties. $E_{2}, E_{5}$ and $E_{8}$ are defined by quadratic equations, giving rise to implicit manifolds with no singular points. Thus they all are differentiable manifolds.

Corollary $2 \mathcal{X}=\left(\bigcup_{i=1}^{17} E_{i}\right)$ is a finite stratification of $\mathcal{X}$.

Proof. Clearly, these sets are disjoint sets and constitute a partition of $\mathcal{X}$. From Theorem 1 they are differentiable manifolds, thus a stratification of $\mathcal{X}$.

Next Table shows the dimensions of the strata above.

\begin{tabular}{|c|c|c|c|c|c|}
\hline Stratum & Dimension & Stratum & Dimension & Stratum & Dimension \\
\hline$E_{1}$ & 8 & $E_{2}$ & 5 & $E_{3}$ & 6 \\
$E_{4}$ & 7 & $E_{5}$ & 5 & $E_{6}$ & 5 \\
$E_{7}$ & 6 & $E_{8}$ & 5 & $E_{9}$ & 4 \\
$E_{10}$ & 6 & $E_{11}$ & 3 & $E_{12}$ & 1 \\
$E_{13}$ & 3 & $E_{14}$ & 4 & $E_{15}$ & 3 \\
$E_{16}$ & 5 & $E_{17}$ & 4 & & \\
\hline
\end{tabular}

\section{Bifurcation diagrams}

A bifurcation diagram of a family of bimodal systems,

$$
\Lambda: \mathbb{R}^{d} \longrightarrow M_{n}(\mathbb{R}) \times M_{n}(\mathbb{R}) \times M_{n \times 1}(\mathbb{R})
$$

is a partition of the parameter space $\mathbb{R}^{d}$ according to the canonical form of the triple of matrices, and induced by the stratification which was given in Section 4 . In particular, this stratification provides the information about which canonical forms are near each other in the sense of local perturbations.

Let us show as an example about how a bifurcation diagram may be obtained.

Example 1 Consider the triple of matrices $\left(\left(\begin{array}{ll}2 & 0 \\ 1 & 3\end{array}\right),\left(\begin{array}{cc}1 & 0 \\ -2 & 3\end{array}\right),\left(\begin{array}{c}1 \\ -1\end{array}\right)\right)$ and the effect of a perturbation on it:

$$
\left(\left(\begin{array}{cc}
2 & \varepsilon_{1} \\
1+\varepsilon_{2} & 3+\varepsilon_{3}
\end{array}\right),\left(\begin{array}{cc}
1 & \varepsilon_{1} \\
-2 & 3+\varepsilon_{3}
\end{array}\right),\left(\begin{array}{c}
1 \\
-1
\end{array}\right)\right) \text {, for small } \varepsilon_{1}, \varepsilon_{2}, \varepsilon_{3} .
$$

If $\varepsilon_{1}=\varepsilon_{2}=\varepsilon_{3}=0$, we obtain the initial triple, which belongs to $E_{2}$. If $\varepsilon_{1}=0, \varepsilon_{3} \neq 0$, we obtain a triple in $E_{4}$. If $\varepsilon_{1}=\varepsilon_{3}=0, \varepsilon_{2} \neq 0$, we obtain a triple in $E_{3}$. Finally, in the case where $\varepsilon_{1} \neq 0$, we obtain a triple in $E_{1}$.

\section{References}

[1] V. I. Arnold, On matrices depending on parameters. Uspekhi Mat. Nauk., 26 (1971). 
[2] M. K. Camlibel, W. P. M. H. Heemels, J. M. Schumacher, Algebraic necessary and sufficient conditions for the controllability of conewise linear systems. IEEE Trans. on Automatic Control, 53 (2008), p. 762-774.

[3] V. Carmona, E. Freire, E. Ponce, F. Torres, On simplifying and classifying piecewise linear systems. IEEE Trans. on Circuits and Systems, 49 (2002), p. 609-620.

[4] V. Carmona, E. Freire, E. Ponce, F. Torres, The continuous matching of two stable linear systems can be unstable. Discrete and continuous dynamical systems 16 (2006), 3, p. 689-703.

[5] M. di Bernardo, C. J. Budd, A. Champneys, P. Kowalczyk, PiecewiseSmooth Dynamical Systems. Springer-Verlag, London (2008).

[6] J. Ferrer, M. D. Magret, M. Peña, Bimodal piecewise linear systems. Reduced Forms. Submitted to Int. J. Bifurcation and Chaos.

[7] E. Freire, E. Ponce, F. Rodrigo, F. Torres, Bifurcation sets of continuous piecewise linear systens with two zones. Int. J. Bifurcation and Chaos, 8 (1998), 11, p. 2073-2097.

[8] E. Freire, E. Ponce, J. Ros, The focus-center-limit cycle bifurcation in symmetric 3D piecewise linear systems. SIAM J. Appl. Math., 65 (2005), 3, p. 1933-1951.

[9] J. E. Humphreys, Linear Algebraic Groups. Graduate Texts in Mathematics, 21, Springer-Verlag, Berlin (1981). 